\title{
VALIDACIÓN DE LA CITOLOGIA CÉRVICO UTERINA CONVENCIONAL CON PRUEBA DE REFERENCIA HISTOPATOLÓGICA EN LA IDENTIFICACIÓN DE CÁNCER ESCAMOCELULAR INVASOR
}

\author{
Ernesto García A. ${ }^{1}$, Julio Díaz P.1, Mario Melo U. ${ }^{1}$, Fabián Parra F.1, Lina Vera C. ${ }^{1}$, Luis \\ Orozco V. ${ }^{1}$
}

${ }^{1}$ Grupo de Investigación en Patología Estructural, Funcional y Clínica, Universidad Industrial de Santander, Bucaramanga, Colombia.

\section{RESUMEN}

Antecedentes: La citología cérvico uterina convencional (CCUC) es la prueba de tamizaje más empleada en la detección del cáncer de cuello uterino, sin embargo su calidad es discutida. Objetivo: Evaluar el rendimiento de la CCUC en el tamizaje de cáncer escamocelular invasor de cuello uterino. Métodos: Fueron seleccionadas 1980 usuarias de la Liga Santandereana de Lucha Contra el Cáncer. Siguiendo un muestreo prospectivo se realizó un análisis estadístico para validar la CCUC en el diagnóstico de carcinoma escamocelular invasor utilizando como prueba de referencia el informe histopatológico de biopsia. Fue determinada la sensibilidad, la especificidad, el valor predictivo positivo (VPP) y negativo (VPN). Resultados: La CCUC tuvo una sensibilidad de $12 \%$, una especificidad de $99 \%$, un VPP de $83,3 \%$ y un VPN de $98,2 \%$ en la identificación de carcinoma escamocelular invasor, en una población con una prevalencia de la enfermedad de $2 \%$. Conclusiones: En este estudio se encontró que la CCUC tuvo unos parámetros de evaluación similares a los patrones internacionalmente definidos. Su baja sensibilidad da a entender que no es un método de tamizaje, por lo cual es recomendable la mejora de los criterios empleados en su interpretación para aumentar este parámetro, así como hacer énfasis en el desarrollo de nuevas tecnologías que permitan un mejor tamizaje y diagnóstico de esta patología, que al igual que la citología convencional ofrezcan su servicio a bajos costos y con facilidad en su implementación.

\section{PALABRAS CLAVES: Citología, rendimiento diagnóstico, estudio de validación}

\section{SUMMARY}

Background: The conventional cervico uterine cytology (CCUC) is the test of screening used in the precocious detection of the uterine cervix cancer, nevertheless its quality is discussed. Objectives: Evaluate the performance of the cytology in the screening of escamocelular invading cancer. Methods: 1980 users of Liga Santandereana de Lucha Contra el Cancer were selected. Following a prospective sampling, a statistical analysis was made to validate the cytology in the diagnosis to escamocelular invading carcinoma using like test of reference the histopathology information of biopsy. Sensitivity, specificity, positive predictive value (PPV) and negative predictive value (NPV) was determined. Results: The cytology had a sensitivity of $12 \%$, a specificity of $99 \%$, a VPP of $83.3 \%$ and VPN of $98.2 \%$ in the identification to escamocelular invading carcinoma, in a population with a prevalence of the disease of $2 \%$. Conclusions: In the present study the cytology had parameters of evaluation similar to the patterns internationally defined. Sensitivity as 
well gives to understand that it is not a screening method because it is not high, thus is recommendable the improvement of the criteria used in his interpretation to increase this parameter, as well as to make emphasis in the development of new technologies that they allow to a better screening and diagnosis of this pathology, that like the conventional cytology offers their service to low costs and with facility in their implementation.

\section{KEY WORDS: Cytology, performance diagnose, validation study}

\section{INTRODUCCIÓN}

El cáncer del cuello uterino es un problema relevante en salud pública con implicaciones trascendentes en la salud de la mujer $(1,2)$, es una causa común de mortalidad y morbilidad en nuestro país $(3,4)$ así como en Latinoamérica (5-7). La identificación precoz es de gran importancia $(8,9)$, ya que es curable en estadios primarios (10-12), lamentablemente en países en vías de desarrollo el $80 \%$ de los casos se hace estados avanzados (13).

La prueba más comúnmente usada en el tamizaje del cáncer de cuello uterino es la Citología Cérvico Uterina Convencional (CCUC), desarrollada por George Papanicolaou en la década de 1930 (14); su implementación ha traído una disminución en la incidencia y mortalidad de esta enfermedad $(8,9)$. A pesar de esto, los resultados no son los esperados, y se han diseñado diferentes métodos para la clasificación de sus resultados $(15,16,17)$ para así mejorar su rendimiento. El protocolo usual de diagnóstico en Colombia es el esquema 1:1:3:3, en el cual se realiza la CCUC cada año durante dos años y luego cada tres años de por vida en las mujeres mayores de 18 años (18). Este tamizaje seriado es realizado con el fin de disminuir el número de falsos negativos que se presentan con esta prueba (19-22), a pesar de lo anterior, un gran número de pacientes con diagnostico de cáncer del cuello uterino, se habían realizado en lapsos cortos de tiempo una CCUC con resultado negativo para malignidad, lo cual se traduce en un aumento sustancial en los costos de salud, pérdida de tiempo valioso en el diagnóstico y tratamiento precoz, como disminución de la expectativa de vida.

El rendimiento de la CCUC en el tamizaje de cáncer de cuello uterino ha sido evaluado en otros lugares del mundo, estas valoraciones han sido ampliamente discutidas (23), debido a que presentan una gran variación en los resultados con sensibilidad de 9,8 (24) a 99\% (25) y especificidad de 24 (26) a 99\% (27), al parecer por formas de evaluación, algunas de las cuales emplean tipos de muestreo y análisis no recomendados (28), es por tanto que existe controversia entre los resultados $(23)$ y en las evaluaciones $(23,29)$. En nuestro país no se encontró que esta metodología haya sido evaluada, por lo que se traza como objetivo de este estudio establecer la validez de la CCUC en la detección de carcinoma invasor de cuello uterino contrastado con prueba de referencia histopatológica.

\section{MATERIAL Y MÉTODO}

Población y muestra. Este estudio fue realizado en la Liga Santandereana de Lucha Contra el Cáncer, Bucaramanga, Colombia, entre los años 2002 y 2003. Las pacientes fueron atendidas inicialmente por el servicio de CCUC, a estas se les realizó examen citológico, interpretado por médicos patólogos con entrenamiento en citopatología, enmascarados con respecto a los datos clínicos de los sujetos del estudio, se clasificaron según la nomenclatura Bethesda $(16,17)$. Luego de 30 a 60 días se siguieron las pacientes con CCUC positiva para carcinoma escamocelular invasor con colposcopia; la clasificación utilizada fue la NIC-OMS (15). Posteriormente se reclasificaron según Bethesda $(16,17)$, lo anterior fue realizado por médicos ginecólogos con entrenamiento en colposcopia y patología de cuello uterino. A continuación fueron sometidas a biopsias colpodirigidas; el material obtenido fue enviado para evaluación histopatológica, por patólogos con entrenamiento en histopatología del cáncer, enmascarados con los datos clínicos y el reporte del estudio citológico, los resultados fueron clasificados según Bethesda $(16,17)$. Además se seleccionó un subgrupo de las citologías negativas para la realización del mismo seguimiento $(29,30)$. Fueron incluidas en el presente estudio a quienes se les realizó biopsia independiente del resultado durante los años que duró el estudio. Fueron excluidos los diagnósticos de ASCUS y AGUS por la falta de correlación entre la prueba evaluada y la utilizada como referencia (Figura 1).

Aspectos éticos. Esta investigación fue rea- 


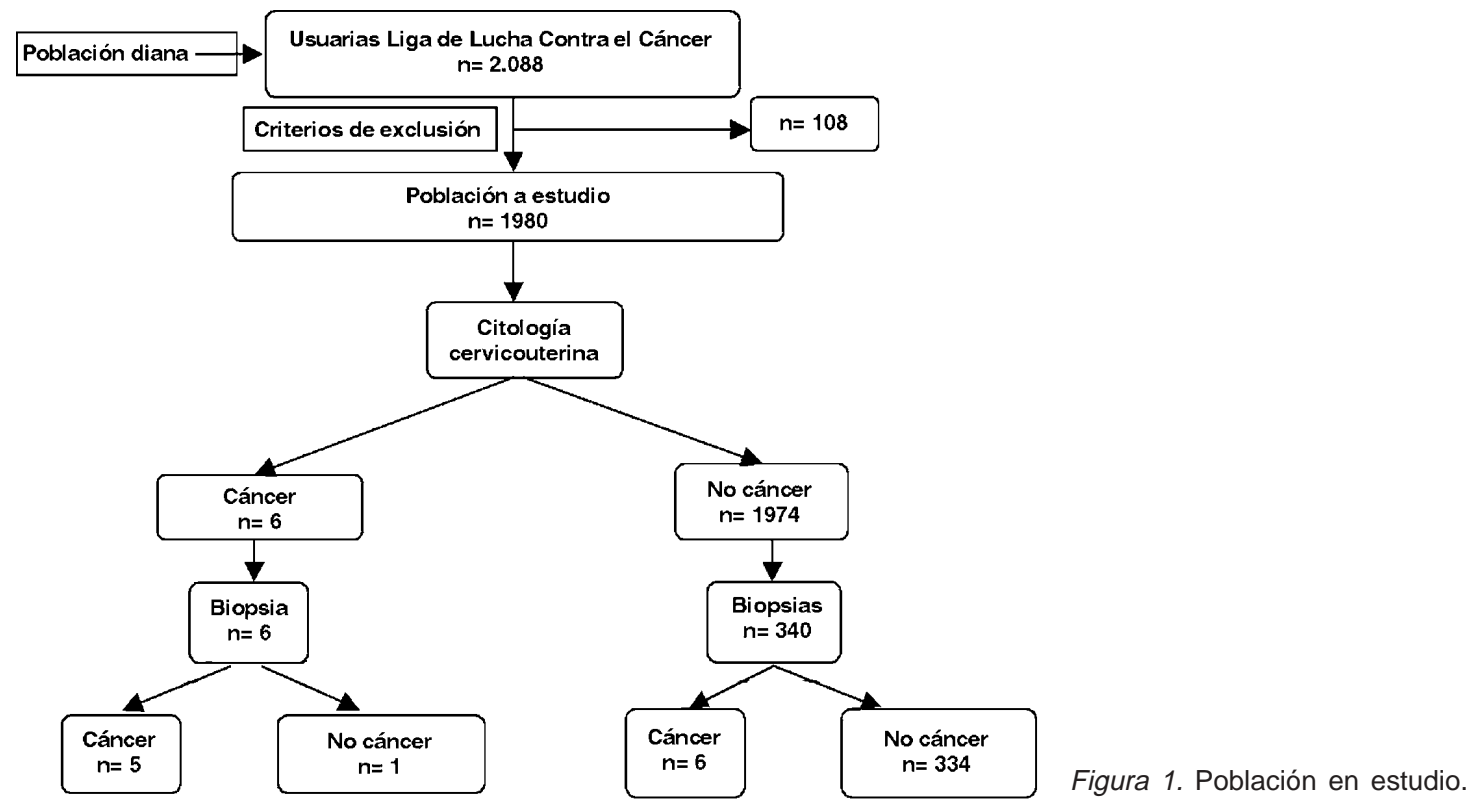

lizada acorde con la legislación nacional (Constitución Política Nacional de 1991 y resolución 008430 de 1993) e internacional (Declaración de Helsinki). Fue aprobada por las directivas de la Liga de Lucha Contra el Cáncer, y catalogada como una investigación sin riesgo. Como examen de referencia se tomó el examen histopatológico de biopsia.

Análisis epidemiológico y estadístico. Se estimo un tamaño de muestra mínimo de 51 muestras en la segunda fase del estudio siguiendo el método de Obuchowsky (31). Se calculó por medio de un muestreo prospectivo $(29,30)$ la sensibilidad, la especificad, el valor predictivo positivo (VPP) y negativo (VPN). Con los valores mostrados en la Figura 1, conformamos la Tabla I.

Teniendo en cuenta el muestreo prospectivo, tenemos en forma directa:

$$
\begin{aligned}
& \mathrm{Q}=\mathrm{VP}+\mathrm{FN} / \mathrm{NO}=(5+1) / 1980=0,00303 \\
& \mathrm{VPP}=\mathrm{VP} /(\mathrm{VP}+\mathrm{FP})=0,83333 \\
& \mathrm{VPN}=\mathrm{VN} /(\mathrm{FN}+\mathrm{VN})=0,98235
\end{aligned}
$$

Reconstruyendo la tabla de contingencia para el tipo de muestreo se puede conformar la Tabla II:

$\mathrm{VP}=\mathrm{Q}$ *VPP

$\mathrm{FN}=(1-\mathrm{Q})(1-\mathrm{VPN})$

$\mathrm{FP}=\mathrm{Q}(1-\mathrm{VPP})$

$F N=(1-Q)$ VPN

Observando la Tabla II se obtiene:

$\mathrm{S}=\mathrm{VP} /(\mathrm{VP}+\mathrm{FN})=0,12$

$\mathrm{E}=\mathrm{VN} /(\mathrm{FP}+\mathrm{VN})=0,99$

Prevalencia $=\mathrm{VP}+\mathrm{FN}=0,02$
Para el manejo estadístico de los datos se utilizaron los programas Epi Info versión Abril 2004 (CDC, USA) (32), y Stata Versión 8.0 (33).

\section{RESULTADOS}

La población estudio fue de 1980 mujeres usuarias de la Liga Santandereana de Lucha Contra el Cáncer, la edad promedio de estas fue de 37,2 años con una DE de 11,1 años. El rendimiento de la CCUC en la identificación de carcinoma escamocelular invasor se presenta en la Tabla III.

\section{DISCUSIÓN}

La prevención y el diagnóstico precoz constituyen las maneras más eficientes en reducir tanto la morbilidad como la mortalidad del cáncer (34). Desde hace años se ha impuesto el método clasificatorio por triage, como una forma acertada en la detección precoz del cáncer. En este método se basa el protocolo de diagnóstico de patología

\section{Tabla I}

TABLA DE CONTINGENCIA 2X2 DE LA PRIMERA FASE DEL MUESTREO

\begin{tabular}{llll}
\hline & & \multicolumn{2}{c}{ Diagnóstico } \\
& & Positivo & Negativo \\
\hline Prueba a evaluar & Positivo & $5(\mathrm{VP})$ & 1 (FP) \\
& Negativo & $6(\mathrm{FN})$ & $334(\mathrm{VN})$ \\
\hline
\end{tabular}


Tabla II

TABLA DE CONTINGENCIA 2x2 DE LA SEGUNDA FASE DEL MUESTREO

\begin{tabular}{llll}
\hline & \multicolumn{2}{c}{ Diagnóstico } \\
& & Positivo & Negativo \\
\hline Prueba a & Positivo & 0,00252 (VP) & $5,347 \mathrm{E}-05$ (FP) \\
evaluar & Negativo & 0,01759 (FN) & 0,97937 (VN) \\
\hline
\end{tabular}

cérvico uterina utilizado mundialmente (18), como en nuestro país (35). En el presente estudio, el diagnóstico de carcinoma invasor encontró que la citología tuvo parámetros de evaluación similares a los patrones internacionalmente definidos para esta prueba los cuales son sensibilidad del $27 \%$ y especificidad del $98 \%$ (13). Hasta la fecha han sido publicados 2 meta-análisis sobre la evaluación del rendimiento de la citología en la identificación de patología oncológica del cuello uterino realizados por Fahay y cols (23) y por Nanda y cols (28), donde los autores expresan la falta de rendimiento de esta prueba en el tamizaje, así como la poca atención a la metodología empleada en el diseño de la mayoría de los estudios de evaluación publicados. Según sus análisis la sensibilidad y especificidad de esta prueba se encuentra entre los rangos de $20-35 \%$ y de $90-95 \%$, respectivamente; los datos aquí encontrados avalan sus apreciaciones. Por otro lado el alto VPN $(98,2 \%)$ indica que, casi siempre, cuando la prueba es negativa la paciente no debería tener enfermedad; y el VPP (83,3\%), igualmente alto, nos permite afirmar que la probabilidad de tener cáncer cuando la prueba es positiva es alta; lo cual confirma la importancia de la prueba como método diagnóstico, pero a su vez da a entender que no es un método de tamizaje por excelencia debido a que no cumple uno de los parámetros establecidos para este tipo de prueba, que es tener una sensibilidad alta (mayor al $80 \%$ ), por lo anterior es recomendable la mejora de los criterios

Tabla III

\section{RENDIMIENTO DE CITOLOGÍA EN EL TAMIZAJE DE} CARCINOMA ESCAMOCELULAR INVASOR

\begin{tabular}{lc}
\hline Parámetro de evaluación & Valor (\%) \\
\hline Sensibilidad & 12,0 \\
Especificidad & 99,0 \\
VPP & 83,3 \\
VPN & 98,2 \\
Prevalencia & 2,0 \\
\hline
\end{tabular}

empleados en su interpretación para aumentar este parámetro, así como hacer énfasis en el desarrollo de nuevas tecnologías diagnósticas que permitan un mejor rendimiento en el tamizaje de esta patología que al igual que la citología convencional ofrezcan su servicio a bajos costos y con facilidad en su implementación.

\section{BIBLIOGRAFÍA}

1. International Agency For Research On Cancer (IARC). Cancer of the cervix. En: IARC. BIENNIAL REPORT 2002-2003. Lyon: IARC. 2004.

2. Waggoner SE. Cervical Cancer. Lancet 2003; 361(9376):2217-25

3. Departamento Administrativo Nacional de Estadística (DANE). Estadísticas Vitales 2003. Disponible en: http://www.dane.gov.co/inf_est/vitales.htm

4. Instituto Nacional de Cancerología, Registro institucional de cáncer del instituto nacional de cancerología, Empresa social del estado. Resultados principales, año 2001. Rev Colombiana Cancerol 2002; 6(3):1-43. Disponible en: http://www.incancerologia. gov.co/revista/v6n3/v6n3a3.pdf

5. Bosch FX, Munoz N, de Sanjose S, Izarzugaza I, Gili M, Viladiu P, Tormo MJ, Moreo P, Ascunce N, Gonzalez LC. Risk factors for cervical cancer in Colombia and Spain. Int J Cancer 1992;11;52(5): 750-8.

6. Arrossi S, Sankaranarayanan R, Parkin DM. Incidence and mortality of cervical cancer in Latin America. Salud Pública Mex 2003;45(Suppl 3):S306-14.

7. Yang BH, Bray FI, Parkin DM, Sellors JW, Zhang ZF. Cervical cancer as a priority for prevention in different world regions: an evaluation using years of life lost. Int J Cancer 2004;109(3):418-24.

8. Manos MM, Kinney WK, Hurley LB, Sherman ME, Shieh-Ngai J, Kurman RJ, Ransley JE, Fetterman BJ, Hartinger JS, Mclntosh KM, Pawlick GF, Hiatt RA.. Identifying women with cervical neoplasia: using human papillomavirus DNA testing for equivocal Papanicolaou results. JAMA 1999;281(17):1605-10.

9. Quinn M, Babb P, Jones J, Allen E. Effects of screening on incidence of and mortality from cancer of cervix in England: evaluation based on routinely collected statistics. BMJ (Clinical Research) 1999; 318(7188):904-8.

10. Saslow D, Runowicz CD, Solomon D, Moscicki AB, Smith RA, Eyre HJ, Cohen C. American Cancer Society. American Cancer Society guideline for the early detection of cervical neoplasia and cancer. CA Cancer J Clin 2002;52(6):342-62.

11. Wright TC Jr, Cox JT, Massad LS, Carlson J, Twiggs LB, Wilkinson EJ. American Society for Colposcopy and Cervical Pathology. 2001 consensus guidelines for the management of women with cervical intraepithelial neoplasia. Am J Obstet Gynecol 2003;189(1): 295-4. 
12. Green JA, Kirwan JM, Tierney JF, Symonds P, Fresco L, Collingwood M, Williams CJ. Survival and recurrence after concomitant chemotherapy and radiotherapy for cancer of the uterine cervix: a systematic review and meta-analysis. Lancet 2001; 358(9284):781-6.

13. World Health Organization. Cervical cancer control in developing countries: Memorandum from a WHO meeting. Bulletin of the World Health Organization WHO Bulletin1996;74(4):345-51.

14. Papanicolaou GN, Traut HF. The diagnostic value of vaginal smears in the carcinoma of the uterus. Am J Obstet Gynecol 1941;42:193-206.

15. Richart RM. Cervical intraepithelial neoplasia. Pathol Annu 1973;8:301-28.

16. National Cancer Institute. The 1988 Bethesda system for reporting cervical/vaginal cytological diagnoses. National Cancer Institute Workshop. JAMA 1989;262:931-4.

17. Smith JH. Bethesda 2001. Cytopathology 2002; 13(1):4-10.

18. Van Niekerk WA, Dunton CJ, Richart RM, Hilgarth $\mathrm{M}$, Kato $\mathrm{H}$, Kaufman $\mathrm{RH}$, et al. Colposcopy, cervicography, speculoscopy and endoscopy. International Academy of Cytology Task Force summary. Diagnostic cytology towards the 21st century: an international expert conference and tutorial. Acta Cytol 1998;42:33-49

19. Spuhler S, Sauthier P. Current indications for colposcopic examination. J Gynecol Obstet Biol Reprod (Paris) 1993;22(7):722-8.

20. Kurman RJ, Henson DE, Herbst AL, Noller KL, Schiffman $\mathrm{MH}$. Interim guidelines for management of abnormal cervical cytology. The 1992 National Cancer Institute Workshop. JAMA 1994;271(23): 1866-9.

21. Allahverdian V, Valaitis J, Kalis O, Pearlman S. Cytology and colposcopy in the diagnosis and management of outpatients with cervical intraepithelial neoplasia. J Reprod Med 1980;24(1):1-4.

22. Sawaya GF, McConnell KJ, Kulasingam SL, Lawson HW, Kerlikowske K, Melnikow J, Lee NC, Gildengorin G, Myers ER, Washington AE. Risk of cervical cancer associated with extending the interval between cervical-cancer screenings. $\mathrm{N}$ England $\mathrm{J}$ Med 2003;349(16):1501-9.

Investigación financiada por el Departamento de Patología de la Universidad Industrial de Santander (UIS).
23. Fahey MT, Irwig L, Macaskill P. Meta-analysis of Pap test accuracy. Am J Epidemiol 1995;141(7): 680-9.

24. Soutter WP, Wisdom S, Brough AK, Monaghan JM. Should Patients with mild atypia in a cervical smear be referred for colposcopy? $\mathrm{Br} J$ Obstet Gynaecol 1986;93(1):70-4.

25. Mannino JR. Natural History of false-negative papanicolau smears: a prospective study using screening colposcopy in addition to cytology. J Am Osteopath Assoc 1998;98(10):542-6.

26. Upadhyay SN, Jha RS, Sinha TK, Mishra NK. Accuracy of cytology in screening for cervical cancer. Indian J Med Res 1984;80:457-62.

27. Davison JM, Marty JJ. Detecting premalignant cervical lesions. Contribution of screening colposcopy to cytology. J Reprod Med 1994;39(5):388-92.

28. Nanda K, McCrory DC, Myers ER, Bastian LA, Hasselblad V, Hickey JD, Matchar DB. Accuracy of the Papanicolaou test in screening for and follow-up of cervical cytologic abnormalities: A Systematic Review. Ann Intern Med 2000;132(10):810-9.

29. Kraemer HC. Evaluating medical tests. Objective and quantitative guidelines. Newbury Park: Sage Publications; 1992.

30. Orozco LC, Camargo DM. Evaluación de tecnologías diagnosticas y tipos de muestreos. Biomédica 1997;17(4):321-24.

31. Obuchowsky N. Sample size calculations in studies of test accuracy. Stat Methods Med Res 1998;7: 371-92.

32. CDC. Division of Public Health Surveillance and Informatics, Epi Info ${ }^{\mathrm{TM}}$ Version 3.2.2 Release Date: April 14, 2004. Disponible en: http://www.cdc.gov/ epiinfo/

33. STATA Corp LP. Statistical Software For Professionals, Version 8.0. 2004. Disponible en: http:// www.stata.com

34. Kiviat NB, Koutsky L. Do our current cervical cancer control strategies still make sense? J Natl Cancer Inst 1996;88(6):317-8.

35. Ministerio de Salud de Colombia. Norma técnica para la detección temprana del cáncer de cuello uterino y guía de atención de lesiones preneoplásicas de cuello uterino, 1999. 